\title{
Non-invasive brain microcurrent stimulation therapy of long-COVID-19 reduces vascular dysregulation and improves visual and cognitive impairment
}

\author{
Bernhard A. Sabel ${ }^{\mathrm{a}, *}$, Wanshu Zhou ${ }^{\mathrm{a}}$, Frank Huber ${ }^{\mathrm{a}}$, Florentina Schmidt ${ }^{\mathrm{a}}$, Kornelia Sabel $^{\mathrm{b}}$, \\ Andreas Gonschorek ${ }^{\mathrm{c}}$ and Mirela Bilc ${ }^{\mathrm{a}}$ \\ ${ }^{a}$ Institute of Medical Psychology, Medical Faculty, Otto-von-Guericke University of Magdeburg, Magdeburg, \\ Germany \\ ${ }^{\mathrm{b}}$ SAVIR-Center, Magdeburg, Germany \\ ${ }^{\mathrm{c}}$ BG Clinic Hamburg, Neurozentrum Hamburg, Germany
}

\begin{abstract}
.
Background: An effective treatment is needed for long-COVID patients which suffer from symptoms of vision and/or cognition impairment such as impaired attention, memory, language comprehension, or fatigue.

Objective: Because COVID-infection causes reduced blood flow which may cause neuronal inactivation, we explored if neuromodulation with non-invasive brain stimulation using microcurrent (NIBS), known to enhance blood flow and neuronal synchronization, can reduce these symptoms.

Methods: Two female long-COVID patients were treated for 10-13 days with alternating current stimulation of the eyes and brain. While one patient (age 40) was infected with the SARS CoV-2 virus, the other (age 72) developed symptoms following AstraZeneca vaccination. Before and after therapy, cognition was assessed subjectively by interview and visual fields quantified using perimetry. One patient was also tested with a cognitive test battery and with a retinal dynamic vascular analyser (DVA), a surrogate marker of vascular dysregulation in the brain.

Results: In both patients NIBS markedly improved cognition and partially reversed visual field loss within 3-4 days. Cognitive tests in one patient confirmed recovery of up to $40-60 \%$ in cognitive subfunctions with perimetry results showing stable and visual field recovery even during follow-up. DVA showed that NIBS reduced vascular dysregulation by normalizing vessel dynamics (dilation/constriction), with particularly noticeable changes in the peripheral veins and arteries.

Conclusions: NIBS was effective in improving visual and cognitive deficits in two confirmed SARS-COV-2 patients. Because recovery of function was associated with restoration of vascular autoregulation, we propose that (i) hypometabolic, "silent" neurons are the likely biological cause of long-COVID associated visual and cognitive deficits, and (ii) reoxygenation of these "silent" neurons provides the basis for neural reactivation and neurological recovery. Controlled trials are now needed to confirm these observations.
\end{abstract}

Keywords: Vision, cognition, restoration, recovery, corona, neuromodulation

*Corresponding author: Bernhard A. Sabel, PhD, Institute of Medical Psychology, Medical Faculty, Otto-von-Guericke University of Magdeburg, Leipziger Straße 44, 39120, Magdeburg, Germany. Tel.: +49391672 1801; Fax: +49391672 1803; E-mail: bernhard.sabel@med.ovgu.de. 


\section{Introduction}

During the worldwide COVID-19 pandemic a large number of initially recovered patients reported different functional complains commonly referred to as "long-COVID" or "post COVID condition" (Guedj et al., 2021). The WHO describes it as a "persistent state of ill health" even of non-hospitalized persons who initially had only mild COVID-19 symptoms but who later progress to symptoms:

"Post COVID-19 condition occurs in individuals with a history of probable or confirmed SARS CoV-2 infection, usually 3 months from the onset of COVID-19 with symptoms that last for at least 2 months and cannot be explained by an alternative diagnosis. Common symptoms include fatigue, shortness of breath, cognitive dysfunction but also others and generally have an impact on everyday functioning. Symptoms may be new onset following initial recovery from an acute COVID-19 episode or persist from the initial illness. Symptoms may also fluctuate or relapse over time" (WHO website 2021).

The main hall-mark of long-COVID that significantly impacts quality of life are cognitive symptoms (Hosp et al., 2021) of attention and executive functions. The number of cases is on the rise. Hampshire et al. (2021) showed that about $3 \%$ of over 12,000 suspected COVID-19 patients developed significant cognitive deficits long after the early infection symptoms had subsided. But the risk is much higher in hospitalized COVID-19 patients, with about $60 \%$ experiencing cognitive decline within four months including impaired speech production, learning, memory and executive functions (Miskowiak et al., 2021), and they can suffer abnormalities in mood such as depression, anhedonia and lower stress resilience (Lamontagne et al., 2021). This is not all that surprising given the bilateral hypometabolism of the brain (Guedj et al., 2021).

Besides impaired cognition, long-COVID patients can also have visual symptoms. However, they are not easily recognized, neither by patients nor by clinicians. They are more benign because of their location in the periphery of the visual field, hence rarely examined. Nevertheless, a systematic review shows that $11.6 \%$ had ocular surface manifestations (Aggarwal et al., 2020). Not only was there viral RNA in the eyes (Bertoli et al., 2020) and retinal vessel abnormalities (Lani-Louzada et al.,
2020), but even $22 \%$ of asymptomatic COVID19 patients had retinal microangiopathy (Landecho et al., 2020), lowered macular vessel and perfusion density (Guemes-Villahoz et al., 2021); and there are cases with acute macular neuroretinopathy (AMN) with paracentral scotomas (Bhavsar et al., 2016, Virgo et al., 2020, Macé et al., 2021). Even vaccination can trigger visual impairments: sudden visual field loss was detected after PfizerBioNTech COVID-19 vaccination (Santovito et al., 2021) (Valenzuela et al., 2021) and AMN paracentral scotomas after AstraZeneca (Mambretti et al., 2021, Bøhler et al., 2021, Book et al., 2021, Drüke et al., 2021).

The question arises as to the pathological mechanisms of this wide array of ophthalmological and neuropsychiatric symptoms. The cardinal problem of Covid-19 (SARS COV 2 virus) is the disturbance of blood vessel health with reduced blood flow (deoxygenation) throughout the body (Buso et al., 2021). We believe that very small micro-vessels (with their relatively large vessel wall surface) are at greatest risk. Two pathological mechanisms, thrombosis and vascular damage, influence each other and amplify vascular dysfunction, especially in the venous outflow system. As we propose below, this insufficient blood flow is the cardinal cause of the wide range of central nervous system symptoms: mild or moderate hypoxia impairs neural activity due to metabolic "silencing" of neurons because of oxygen (and glucose?) deprivation. Now neurons can no longer fire action potentials, reducing the flawless interactions of neural communication in a highly dynamic brain functional connectivity network. In fact, healthy mental function depends on a healthy "brain spacetime", i.e. high speed adaptations of neural networks in the msec. range (Wu \& Sabel, 2021). Metabolic (oxygen) deprivation is expected to slow down this neural processing and thus disturb fast networks interactions, impacting neural synchronization and integration. It is the dynamics of the brain network in the msec.range which determines if functions are normal or abnormal. Hence, if blood flow is not properly regulated, functional impairments are expected such as longer reaction times or greater difficulties in coordinating complex tasks (Bola et al. 2015a,b, Wu \& Sabel 2021).

Long-COVID is on the rise and no effective treatment exists yet to improve visual and cognitive impairments. While different biological mechanisms of long-COVID have been discussed (Proal \& VanElzakker 2021), physiotherapy and rehabilitation 
are currently the only approaches to reduce symptoms, but they take weeks or months, with less than satisfactory improvement. Hence, a method is needed to address the root cause of restoring blood flow regulation in the eye and brain and/or improve the synchronization of brain functional connectivity networks (Nurek et al., 2021).

Neuromodulation with non-invasive brain stimulation using microcurrents (NIBS) is one option to accomplish this goal. NIBS can be applied locally to enhance or suppress specific neurological function such as locomotor behavior (Kang et al., 2020), depression (Kisely et al., 2018), or attention (Westwood et al., 2021). Most relevant for the present study is that NIBS can enhance visual (for review Sabel et al., 2020b) and cognition dysfunctions (Siegert et al., 2021, Teselink et al 2021). Possible mechanisms of NIBS include (i) alterations of excitability of neuronal tissue (excitation or inhibition), (ii) interference of brain oscillations, (iii) reorganization of brain functional connectivity networks, and (iv) stimulation of blood flow (Sabel et al., 2020a, b).

A growing number of studies of the visual system showed that NIBS is able to (i) reactivate hypometabolic "silent neurons" (Sabel et al., 2020b); (ii) increase expression of neurotrophic factors which promote neuroprotection and synaptic plasticity; (iii) enhance brain network reorganization (Bola et al., 2014; Wu \& Sabel 2021), and (iv) improve blood flow and vascular autoregulation (Sabel, Flammer, Merabet 2019). In fact, vascular dysregulation produces signs and symptoms, collectively referred to as the "Flammer syndrome" (Konieczka. \& Flammer 2016, Golubnitschaja 2019), which can affect many organs, especially the eye, brain and inner ear.

In our lab we typically use transorbital alternating current stimulation (tACS) to improve vision in low vision patients. It has a "double-punch effect": on the one hand, it forces neurons to fire action potentials, and - on the other hand - it enhances blood flow. It is this "double-punch" effect which could explain (partial) restoration of disturbed neural networks in the brain with clinical benefits of improving vision (Gall et al., 2016) and enhancing"brain spacetime", i.e. very fast network reorganization (Wu \& Sabel 2021) (see discussion).

Considering that visual and cognitive symptoms in long-COVID may also be a neuro-vascular "tandem" problem of reduced blood flow (vascular dysregulation, deoxygenation) with associated neural hypometabolism, we hypothesized that the tACS "double-punch" therapy might be able to reduce
long-COVID symptoms. As we now show in two patients, a 10 to 13 days NIBS treatment can achieve a remarkably fast and long-lasting recovery of visual fields and cognitive functions, the extent and speed of which were surprising.

\section{Methods}

\subsection{Clinical case description}

This is a clinical analysis of two patients, K.H. and G.B., who visited our SAVIR-Center in Magdeburg to receive treatment for their vision impairment according to existing SOPs. Data were collected during routine testing and, per request of patient K.H., additional test in the BG Clinic-outpatient service center in Hamburg.

Patient K.H. is a 40-years old female German citizen who works as a health data processing manager. Her medical history includes migraines since age of $18 \mathrm{yrs}$, but no other remarkable events and no current medication. She lives an active lifestyle, enjoys working and riding the bike regularly. Both the patient and her husband were diagnosed with COVID-19 in December 2020, but due to a mild course of the disease, hospitalization was not needed. Symptoms included headaches and low level of fever which recovered during 14 days of quarantine, no coughing, no muscle pain. After an additional 2 weeks of holidays, she returned to work for 2 days.

Patient G.B. is a 72-years old female British citizen that runs a successful holiday letting business. Being a former prima ballerina, she has an active lifestyle, goes for long walks and practices Pilates regularly. She does not smoke and consumes little, if any, alcohol. Her medical history revealed no significant medical conditions and the patient reported no current medication. In February 2021 she was vaccinated with AstraZeneca. During the following weeks she reported having had strong headaches, loss of vision in the left eye, loss of balance as well as cognitive impairments. As symptoms continued, she consulted her physician for an ophthalmological exam with the diagnosis of a left paracentral acute middle maculopathy, negative uveitis screen, negative inflammatory markers, negative carotid dopplers. She was prescribed prednisolone $(60 \mathrm{mg} /$ day for one week, then $50 \mathrm{mg}$ for the next three weeks). Follow-up head MRI indicated age related involutional change with 1 or 2 nonspecific foci of white matter signals, but no evidence of hemorrhage, infarct 
or space occupying lesions. MRV venogram indicated no evidence of venous sinus or cortical venous thrombosis.

\subsection{NIBS treatment}

Patient K.H. and G.B. received 13 and 10 therapy sessions, respectively, of transcranial alternating current stimulation (tACS) at the SAVIR-Center (www.savir-center.com) using the CE-certified SASm-neuromodulation device (SAVIR GmbH, Berlin, Germany). Each session lasted 30-45 min. where currents with density $<2$ mAmp were delivered to the eyes and brain while the patient was sitting comfortably on a chair, with electrodes positions near the forehead. Each session was followed by a resting period of around $15 \mathrm{~min}$, while the patient had her eyes covered with a warm mask. Both patients tolerated the treatment well, and no adverse or serious adverse events were noted. In line with the "holistic" SAVIR therapy (SAVIR-Center, Magdeburg, Germany), the patient also received psychological counseling and learned relaxation techniques.

\subsection{Neurovisual and cognitive assessment}

Patient K.H.: visual fields were assessed with the Oculus Twinfield static perimetry (area $6,0^{\circ}-70^{\circ}$, Fast threshold, Stim III, Wetzlar, Germany) approx. 2 weeks before and 2, respectively 3 weeks after NIBS therapy (Fig. 1). At her request, K.H. was also formally tested for her cognitive abilities in the BG Clinical Center (Hamburg) before and after therapy. Here, two cognitive tests were applied to quantify attention, verbal learning and verbal memory using the "Test of Attentional Performance" (TAP, Germany version: "Testbatterie zur Aufmerksamkeitsprüfung", Version 2.3.1) and the "Auditory Verbal Learning Test (AVLT)" (German Version: "Verbaler Lern- und Merkfähigkeitstest", VLMT) (Table 1).

Patient G.B.: visual fields were obtained with the Humphrey Field Analyzer (HFA; central 30-2, SITA-Standard, Stim III, Carl Zeiss Meditec, Jena, Germany) (Fig. 2) and high-resolution perimetry (HRP) (NovaVision, Magdeburg, Germany) before and after therapy.

\subsection{Vascular dysregulation assessment}

DVA recording of vessel dynamics in the retina and data collection: In patient K.H. we measured vessel

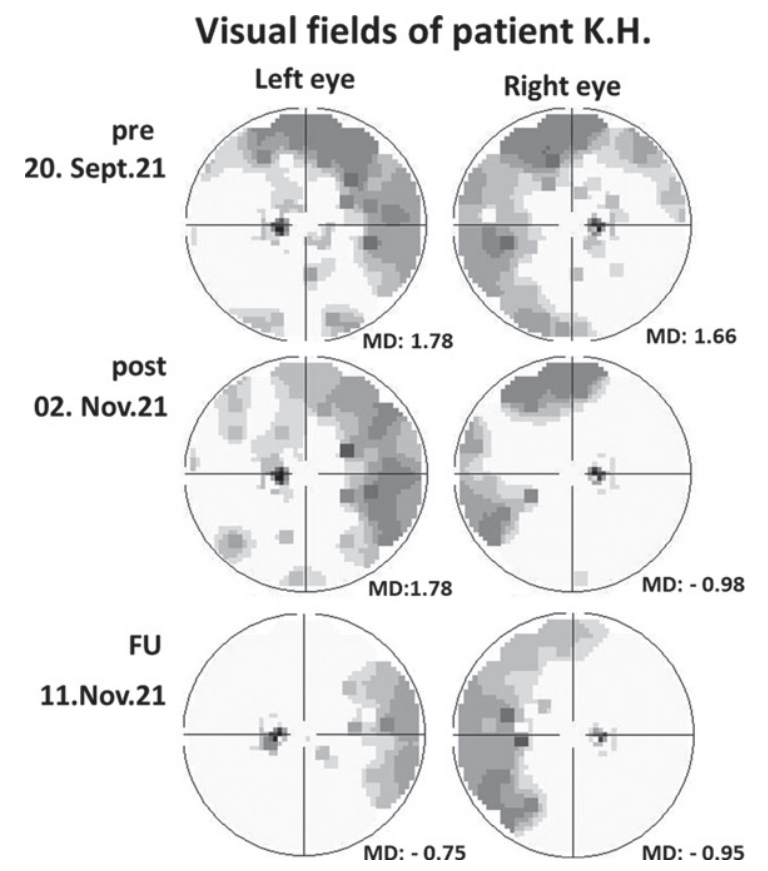

Fig. 1. Recovery of 75-degree Oculus visual field of each eye in patient K.H. following NIBS treatment. Dark areas represent regions of vision loss. They are located in nasal visual field in both eyes and improve following treatment, with additional recovery at follow-up. (MD = mean deviation. Note that positive values indicate the patient is worse than her age norm; negative values indicate that the patient is better then her age group).

dynamics in the retina using the Dynamic Vascular Analyzer (DVA) before and after treatment (Figs. 3, 4). Unlike the angio-OCT, the DVA can differentiate the vessel dilation responses as a function of neuronal activity. Neural activity is triggered by flickering light pulses, and the so induced vessel response can be interpreted as a surrogate marker of vascular dysregulation in the brain.

With the DVA we video-recorded vessel diameters before, during, and after flicker light stimulation to quantify the retinal vessel dilation response which informs us of arterial and venous vessel health. Because the retina is central nervous system (CNS) tissue, i.e. with brain-like neurons and vasculature, the DVA can be considered a surrogate biomarker of vascular health of the brain. In fact, DVA revealed already abnormalities in different CNS diseases, including Alzheimer Disease (Querques et al., 2019) and primary open angle (POAG) and normal tension glaucoma (NTG) (Gugleta et a. 2012).

To measure vessel dynamics, the retina was video imaged using a fundus camera (Zeiss, Jena, Germany) at $30^{\circ}$ field of view. The fundus camera was 


\section{Visual fields of patient G.B.}

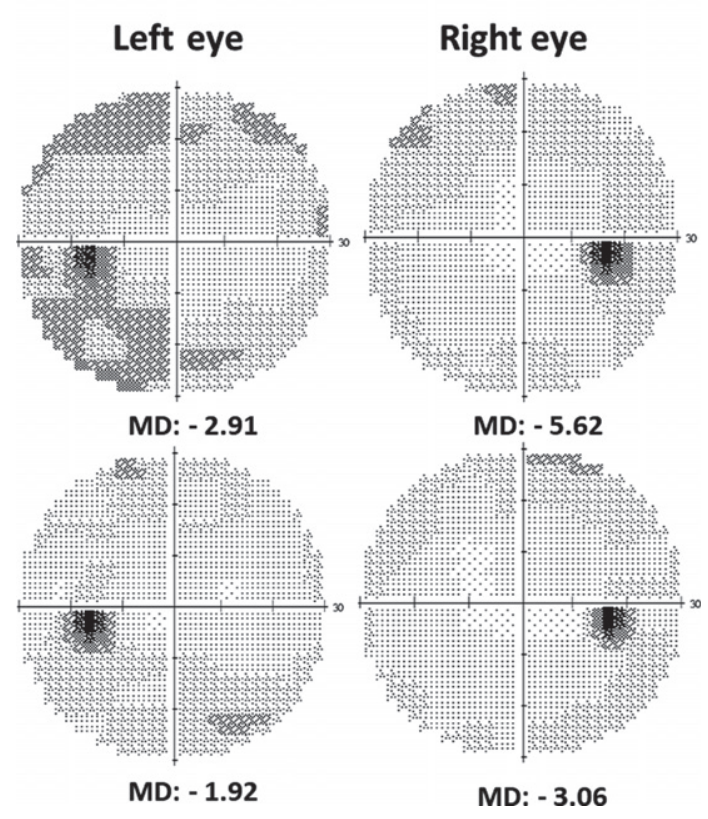

Fig. 2. Recovery of 30-degree Humphrey visual field in patient G.B. following NIBS treatment $(\mathrm{MD}=$ mean deviation: lower negative values (i.e., closer to zero) indicate more vision in this perimetry).

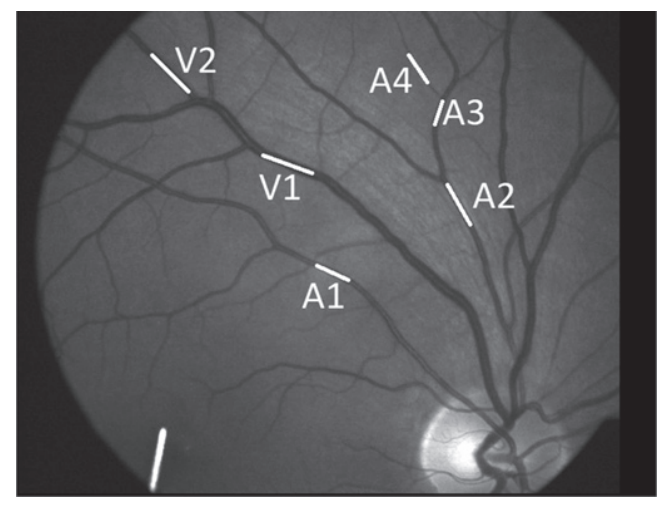

Fig. 3. Example of a retinal image in Patient K.H. The DVA analysis program quantifies the absolute vessel diameter in MU (measurement unit) using the Gullstrand's eye as a template. The average of three 30 s recording just prior to every flicker light was taken as the baseline value (100\%) for the analysis of the evoked dilation response. Larger and smaller arteries and veins at different branch orders: first (A1, V1), second (A2, V2), third (A3) and fourth order (A4). Because the DVA images focus mostly on the central retina, only few $3^{\text {rd }}$ or $4^{\text {th }}$ order vein branches can be measured.

connected to the DVA-analysis system (DVA 2.0, Imedos, Jena, Germany). To obtain a full image of the patient's central retina, the pupils were dilated

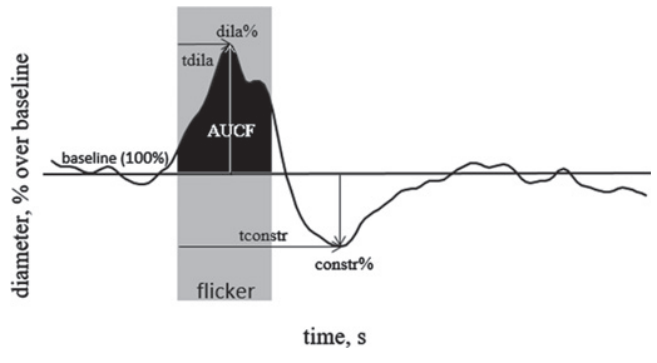

Fig. 4. The parameters of retinal vascular response to flicker are as follows: "maximal dilation" (dila\%): peak dilatation during flickering period compared to baseline; "time to maximal dilation" (tdila): time to peak dilatation after flickering onset; "maximal constriction" (constr\%): peak constriction after flicker onset compared to baseline; "time to maximal constriction" (tconstr): time to reach peak constriction; "area under the curve during flicker stimulation" (AUCF): area under the response curve during the flickering period.

with Mydriatics ( $1 \mathrm{ml}$ solution of $5.0 \mathrm{mg}$ of tropicamide, Pharma Stulln GmbH, Stulln, Germany), and the retina of each eye was then imaged and video recorded during a 5-6 min session for each eye which consisted of three measurement periods: (i) a $50 \mathrm{sec}$. baseline measurement, (ii) three repeated diffuse luminance $12.5 \mathrm{~Hz}$ flicker stimulation periods of $20 \mathrm{sec}$. each, and (iii) a subsequent $80 \mathrm{sec}$. postflicker period. The three $20 \mathrm{sec}$. flicker stimulation periods were then averaged and the video was analysed for vessel diameter changes over time in the upper and lower retinal sector to assess vessel dilation and vasoconstriction dynamics (Seifert et al., 2002, Garhofer et al., 2010). In a healthy retina, the light flickering activates retinal neurons which then provokes vessel dilation through neurovascular coupling.

The vessel diameter before, during and after light flickering show the dilation capacity of the vessels of interest which was then analysed using different parameters as a function of the branch order in the vascular tree (Tables 2 and 3). To carry out statistical analysis, each vessel was treated as an independent data point (19 arteries, 17 veins). Measurements were only taken if the respective vessel had sufficient contrast to the surrounding fundus with no crossing or bifurcation in the measuring segment, and with a curvature $<30^{\circ}$. The raw data of all vessels was then saved for further analysis. The DVA metrics are displayed in Fig. 4. Paired sample $t$-tests were conducted to investigate changes in DVA parameters, before and after therapy for all the measured blood vessels and separately for central and peripheral ones. 


\section{Results}

\subsection{Clinical and subjective observations}

\subsubsection{Patient K.H.}

K.H. subjective complaints before therapy: approximately four weeks after her COVID infection in December 2020, K.H. reported an episode of not feeling well, having lost strength and not being able to get out of bed. The symptoms continued in January with the patient having a constant feeling of weakness, not being able to go for walks anymore, having difficulty even with small activities like showering or washing her hair, and she had a strong sleeping urge (up to $18 \mathrm{hrs} / \mathrm{day}$ ). In the next months, K.H. had trouble doing the housework (e.g. could not cook anymore or do the typical household chores). She noticed severe cognitive deficits: being forgetful, feeling overwhelmed, had difficulties finding words, holding conversations, or doing multiple tasks simultaneously. She also noticed changes in her vision, episodes of occasionally losing acuity (foggy vision) and being unable to focus anything even for very short periods of time. These visual impairments were rather short, lasting only a few minutes at a time. In June 2021, she began a six week rehabilitation program that included computer-based cognitive rehabilitation training, relaxation therapy (progressive muscle relaxation and breathing exercises), occupational therapy, as well as music and dance therapy. However, rehabilitation did not help her cognitive abilities much, but she learned how to relax and better cope with her situation (increased acceptance, less self-blaming).

During her first consultation in mid-September 2021, the patient reported still having difficulties finding words, slowed reaction time, short-term memory deficits (e.g., forgets the beginning of the sentence while reading), attention deficits (e.g., not being able to concentrate on one among multiple stimuli), and she did not feel confident enough to drive her car anymore. She still had sudden episodes when her vision was unclear and unfocused. These episodes were not occurring as often as before, but remained a continuous source of concern.

K.H. subjective recovery after therapy: after NIBS therapy, K.H. subjectively reported having no more episodes of sudden visual acuity loss, and she noticed significant improvements of her mental state already after the third therapy session. This consolidated during the remaining time of the therapy ("The brain fogginess was suddenly gone and my mind is clear again"). She was able to engage in conversations again, did no longer get so easily distracted by simultaneous stimuli, was now able again to focus her attention on particular aspects of the situation, and she felt able again to make plans and follow them through. And she could attend to her child and household tasks with no more difficulties in carrying out everyday tasks, and she started going back to work. This recovery was stable as of Nov. 27, 2021.

\subsubsection{Patient G.B.}

G.B.'s subjective complaints before therapy: at her first consultation mid-Sept 2021, i.e. before NIBS, G.B. reported difficulties concentrating and focusing her attention, word-finding difficulties, short-term memory difficulties, and not being able to work any longer. She described her vision in the right eye as being normal, but unclear "broken-up" in her left eye. She felt unsafe to walk around.

G.B.'s subjective recovery after therapy: After therapy G.B. subjectively noticed improvements in her vision, reporting a clearer picture and an enlarged peripheral visual field ("I am able to see everything around me which is life changing") and that her eyes were now "working together again". G.B. also felt to have improved cognitively: she was more comfortable to walk around, and reading was not as tiring as before. She felt much more alert and brighter, could now carry-on conversations with her children again, could remember what she was just doing before, no longer struggling for words, and being able to plan and to remember things better. She felt ready to go back to work in her business. In sum, she felt a lot more positive which was a "huge blessing" for her.

\subsection{Quantification of visual field recovery}

Both patients had intact central but impaired peripheral field defects. In the description below, note that visual field defects are represented by positive mean deviation values (MD) in Oculus perimetry of patient (K.H.) while negative values in Humphrey perimetry of patient (G.B.). Both patients showed some recovery in their visual field with different dynamics as now described:

Patient K.H.: Visual fields recovered noticeably in the right eye, where mean sensitivity (MS) and mean defect (MD) at the three time points (pre-, 2 wks post- and 3 wks post- therapy) were as follows: $\mathrm{MS}=13.92 \mathrm{~dB}, 16.56 \mathrm{~dB}$ and $16.53 \mathrm{~dB} ; \mathrm{MD}=1.66$, -0.98 and -0.95 . In the left eye, no changes were observed two weeks after the therapy, with values 
Table 1

Cognitive functioning pre vs. post ACS treatment in patient K.H.

\begin{tabular}{lccc}
\hline & Before & After & $\%$ change \\
\hline Attention (TAP) & & & \\
Alertness & & & \\
$\quad$ Median RT without signal & $854 \mathrm{msec}$ & $555 \mathrm{msec}$ & $35.01 \%$ \\
$\quad$ Median RT with signal & $746 \mathrm{msec}$ & $508 \mathrm{msec}$ & $31.90 \%$ \\
Go/NoGo & & & \\
$\quad$ Median RT (errors) & $800 \mathrm{msec}(3)$ & $617 \mathrm{msec}(0)$ & $22.88 \%$ \\
Divided attention & & & \\
$\quad$ Median RT auditory & $1408 \mathrm{msec}$ & $835 \mathrm{msec}$ & $40.70 \%$ \\
$\quad$ Median RT visual & $1332 \mathrm{msec}$ & $887 \mathrm{msec}$ & $33.41 \%$ \\
Executive functions (TAP) & & & \\
Flexibility (alternating verbal) & $1961 \mathrm{msec}$ & $775 \mathrm{msec}$ & $60.48 \%$ \\
$\quad$ Median RT & 11 & 3 & $72.72 \%$ \\
$\quad$ Errors & & & $-21.29 \%$ \\
Working Memory (level 3)* & & & \\
$\quad$ Median RT (errors) & 1104 msec (1) & 1339 msec (4) \\
Memory (VLMT list A) & 7 words & 8 words & $14.29 \%$ \\
Supraspan & 46 words & 50 words & $8.70 \%$ \\
Learning capacity & 7 words & 8 words & $14.29 \%$ \\
Retrieval Interference List I & 7 words & 9 words & $28.57 \%$ \\
Retrieval after interference & 2 words & 2 words & $0 \%$ \\
Retroactive interference & 7 words & 7 words & $0 \%$ \\
Delayed retrieval & 2 words & 3 words & $50 \%$ \\
Medium term remembering & 10 words & 9 words & $-10 \%$ \\
Recognition & & &
\end{tabular}

Note $:$ RT $=$ reaction time in $\%$ change for RT and errors $=($ pre - post $) /$ pre $\times 100$. Change in word count in $\%=($ post - pre $) /$ pre $\times 100 .{ }^{*}$ Patient reported to be overly tired during the post-test.

remaining stable for MS (13.8 dB) and MD (1.78). During follow-up, however, visual field improvements were noted. Now MS for the left eye was $16.33 \mathrm{~dB}$ and MD was -0.75 (Fig. 1).

Patient G.B.: Visual Field Index (VFI) showed no central but peripheral visual field deficits with improvements from $97 \%$ to $99 \%$ in the right eye and from $92 \%$ to $95 \%$ in the left eye. Mean deviation values decreased from $-2.91 \mathrm{~dB}$ to $-1.92 \mathrm{~dB}$ in the right eye and from $-5.62 \mathrm{~dB}$ to $-3.06 \mathrm{~dB}$ in the left eye (approaching the age norm). Computer-based high-resolution perimetry confirms improvement: detection accuracy slightly increased from $95.42 \%$ to $96.83 \%$ in the right eye and from $93.66 \%$ to $96.48 \%$ in the left eye, with improved mean reaction time pre/post for the right $(411 / 380 \mathrm{~ms})$ and left eye $(445 / 411 \mathrm{~ms})$. Visual acuity measures $(5 \mathrm{~m}$, without correction) indicated improvements for both eyes. While testing the right eye before the treatment, the patient could only distinguish light and shapes on the visual chart, but after NIBS we were able to measure the visual acuity at a value of 0.25 (decimal). In the left eye visual acuity improved from 0.42 to 0.75 . MARS letter test of contrast sensitivity (Oculus, Wetzlar, Germany) showed normal contrast sensitivity with small improvements for the binoc- ular measures (pre/post $=1.64 / 1.76$ ) and monocular left eye (pre/post $=1.68 / 1.72)$, with values remaining unchanged for the right eye (1.68) (Fig. 2).

\subsection{Quantification of cognitive recovery}

K.H. showed improvements in almost all cognitive domains (Table 1). For interpretation of results, patient's values were compared with healthy, age-similar and educationally equivalent persons (percentile ranks (PR) normal range 16-84).

Both the intrinsic alertness (tonic alertness) and the phasic alertness improved after the therapy, but it still remained far below the norm (PR in both $<1$ ). The ability to react easily under stimulus selection conditions and the ability to inhibit reactions also improved after the therapy by $23 \%$, which was still below norm (PR 1). The error rate was clearly improved from the first ( 3 errors, PR 18) to the second appointment (0 errors, PR > 14). Clear improvements were also observed for divided attention, which is the ability to process visual and auditory information in parallel. While, the reaction to auditory stimuli continued to be rated as below average for both measures (PR 1), the reaction to visual stimuli after therapy reached the normal range (PR 21). Furthermore, cognitive flex- 


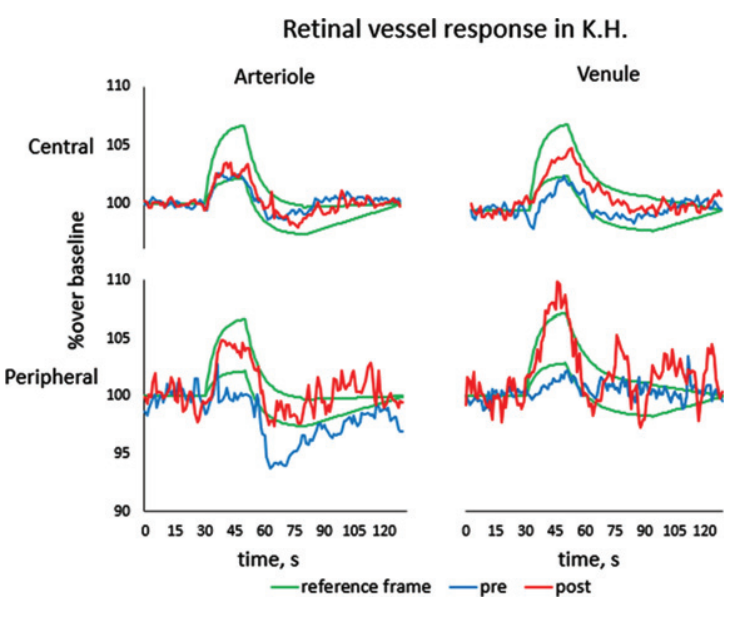

Fig. 5. Central and peripheral retinal vessel dilation and constriction response to flicker light in DVA before and after NIBS in patient K.H. Recovery of vascular dysregulation was absent in the central (large) artery but present in the central vein and in both peripheral vessels; recovery was most pronounced in the peripheral (smaller) vein. Note that the peripheral artery, not the vein, shows a constriction despite lack of prior dilation.

ibility greatly improved, with patient's performance changing from below average to normal after therapy, both in terms of reaction time (PR 1 vs. PR 34) as well as error rate (PR 7 vs. PR 54). One measure was worse after therapy, but patient reported having been very tired, almost falling asleep during this last task of the test battery. In her judgement this negative result fails to reflect her maximum ability because her working memory did not worsen but actually had improved. Improvements were also noticed on the verbal-episodic memory. Both before and after treatment the immediate word span and learning capacity were classified in the normal average range, while retrieval after interference, delayed retrieval and recognition, though improved, were still classified as below average.

\subsection{Recovery from vascular dysregulation}

As displayed in Figs. 5-6, the vascular autoregulation parameters in patient K.H. were markedly impaired before treatment. Central arterial and venous dilation dynamics (large vessel) was at or below the lower limit of the reference window of healthy subjects (Fig. 5), whereas the smaller peripheral vessels, both arteries and veins, were even lower. Interestingly, the peripheral artery fails to dilate, yet it still constricts at the post-flicker time point. This might suggest that the nature of the dysregulation is not only a failure to dilate, but a dilation-independent
NIBS effect on vessel dilation in K.H.

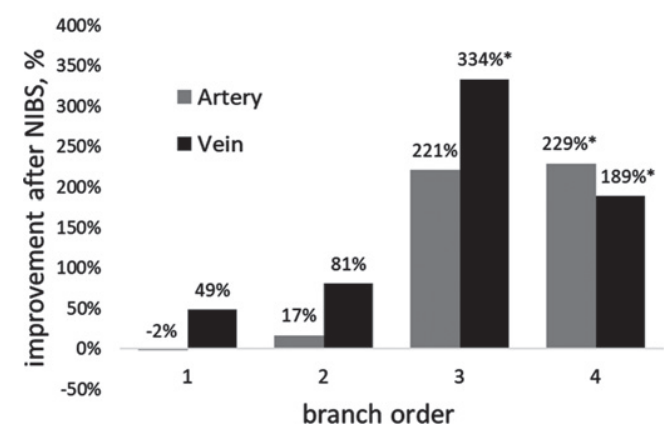

Fig. 6. Percent change over baseline of maximal dilation after NIBS treatment as a function of branch order of arteries and veins. Branches of lower order are smaller than branches of higher order. $\%$ improvement $=($ post-pre $) /$ pre $\times 100 .{ }^{*}$ Note that the samples size of third order vein, fourth order artery and vein are only $2,2,1$.

(paradoxical) constriction which reduces blood flow far below normal levels, a double-punch problem for the small arteries. After NIBS treatment we observed a remarkable recovery of vessel dynamics, and the change was particularly noted in the peripheral arteries and veins, where dilation and constriction capacity of both improved by more than $300 \%$ in some parameters. In the peripheral vein the dilation exceeded the normal reference window at follow-up (see Fig. 5 and Tables 2, 3). In sum, our observation post-treatment shows that NIBS improves vessel dynamics (dilation/constriction) to normal levels. When viewed together with the visual field findings, both subjective and objective visual field improvements continued in the post-treatment time period, and DVA confirms that NIBS normalizes vessel autoregulation.

\section{Discussion}

Because there is no effective treatment of longCOVID patients' visual and cognitive impairments, we tested the therapeutic effects of NIBS to explore if a short-term, 10-day treatment could reduce the long-COVID symptoms and recover some vision, attention, memory, language comprehension, and/or fatigue. We reasoned that since NIBS is known to enhance blood flow, it might also be effective to reduce vascular dysregulation, thereby improving neuronal synchronization of brain functional connectivity networks and achieving some recovery from the long-COVID symptoms. In both of our cases, clear recovery was already noted on days $3-4$, the extent and speed of which was rather surprising. 
Table 2

Average retinal arteriole response pre vs. post ACS treatment

\begin{tabular}{|c|c|c|c|c|}
\hline & pre & post & $\%$ change & $p$-value \\
\hline \multicolumn{5}{|l|}{ All arteries } \\
\hline vessel number & 19 & 19 & & \\
\hline Absolute diameter, MU & 96.6 & 94.7 & $-2 \%$ & 0.023 \\
\hline Mean maximal vessel dilation, $\%$ over baseline & 2.8 & 4.8 & $71 \%$ & 0.018 \\
\hline Time of maximal vessel dilation, second & 12.1 & 14.8 & $6 \%$ & $\mathbf{0 . 0 5 3}$ \\
\hline Mean maximal vessel constriction, $\%$ over baseline & -3.4 & -2.6 & $-24 \%$ & 0.14 \\
\hline Time of maximal vessel constriction, second & 37.9 & 38 & $0 \%$ & 0.99 \\
\hline Area under the curve during flicker light, $\%$ x second & 31.5 & 54.5 & $73 \%$ & 0.033 \\
\hline \multicolumn{5}{|l|}{ Central arteries } \\
\hline vessel number & 11 & 11 & & \\
\hline Absolute diameter, $\mathrm{MU}$ & 106.9 & 105 & $-2 \%$ & 0.16 \\
\hline Mean maximal vessel dilation, $\%$ over baseline & 3.6 & 4 & $11 \%$ & 0.69 \\
\hline Time of maximal vessel dilation, second & 13.3 & 15.5 & $5 \%$ & 0.29 \\
\hline Mean maximal vessel constriction, $\%$ over baseline & -2.7 & -2.4 & $-11 \%$ & 0.68 \\
\hline Time of maximal vessel constriction, second & 34.6 & 38.9 & $7 \%$ & 0.40 \\
\hline Area under the curve during flicker light, $\%$ x second & 44.5 & 47.6 & $7 \%$ & 0.83 \\
\hline \multicolumn{5}{|l|}{ Peripheral arteries } \\
\hline vessel number & 8 & 8 & & \\
\hline Absolute diameter, MU & 82.5 & 80.5 & $-2 \%$ & 0.028 \\
\hline Mean maximal vessel dilation, $\%$ over baseline & 1.8 & 6 & $233 \%$ & 0.000 \\
\hline Time of maximal vessel dilation, second & 10.4 & 13.9 & $9 \%$ & $* 0.08$ \\
\hline Mean maximal vessel constriction, $\%$ over baseline & -4.4 & -2.9 & $-34 \%$ & $* 0.08$ \\
\hline Time of maximal vessel constriction, second & 42.5 & 36.8 & $-8 \%$ & 0.60 \\
\hline Area under the curve during flicker light, $\%$ x second & 13.7 & 64 & $367 \%$ & 0.000 \\
\hline
\end{tabular}

$\%$ change $=($ post-pre $) /$ pre $\times 100 \% ;$ All arteries $=$ all artery branches in retinal image; Central artery $=$ first and second branches, Peripheral artery $=$ third and fourth branches; two-sided paired $t$-test $p$-values; ${ }^{*}=$ trend.

Table 3

Average retinal venule response pre vs. post ACS treatment

\begin{tabular}{|c|c|c|c|c|}
\hline & pre & post & $\%$ change & $p$-value \\
\hline \multicolumn{5}{|l|}{ All veins } \\
\hline vessel number & 17 & 17 & & \\
\hline Absolute diameter, MU & 119.4 & 116.1 & $-3 \%$ & 0.001 \\
\hline Mean maximal vessel dilation, $\%$ over baseline & 2.3 & 4.9 & $113 \%$ & 0.001 \\
\hline Time of maximal vessel dilation, second & 15.9 & 16.5 & $1 \%$ & 0.49 \\
\hline Mean maximal vessel constriction, $\%$ over baseline & -2.4 & -2.1 & $-13 \%$ & 0.64 \\
\hline Time of maximal vessel constriction, second & 46.3 & 61.5 & $20 \%$ & 0.17 \\
\hline Area under the curve during flicker light, \%x second & 20.7 & 48.1 & $132 \%$ & 0.002 \\
\hline \multicolumn{5}{|l|}{ Central veins } \\
\hline vessel number & 14 & 14 & & \\
\hline Absolute diameter, MU & 126.5 & 122.3 & $-3 \%$ & 0.000 \\
\hline Mean maximal vessel dilation, $\%$ over baseline & 2.3 & 3.9 & $70 \%$ & 0.000 \\
\hline Time of maximal vessel dilation, second & 16.4 & 16.6 & $0 \%$ & 0.81 \\
\hline Mean maximal vessel constriction, $\%$ over baseline & -2.7 & -2.1 & $-22 \%$ & 0.46 \\
\hline Time of maximal vessel constriction, second & 37.9 & 62.1 & $36 \%$ & 0.044 \\
\hline Area under the curve during flicker light, $\%$ x second & 20.1 & 35.7 & $78 \%$ & 0.000 \\
\hline \multicolumn{5}{|l|}{ Peripheral veins } \\
\hline vessel number & 3 & 3 & & \\
\hline Absolute diameter, MU & 86.3 & 87 & $1 \%$ & 0.54 \\
\hline Mean maximal vessel dilation, $\%$ over baseline & 2.4 & 9.6 & $300 \%$ & $* 0.06$ \\
\hline Time of maximal vessel dilation, second & 13 & 16 & $7 \%$ & 0.53 \\
\hline Mean maximal vessel constriction, $\%$ over baseline & -1.3 & -2.4 & $85 \%$ & 0.25 \\
\hline Time of maximal vessel constriction, second & 85.7 & 58.7 & $-23 \%$ & 0.29 \\
\hline Area under the curve during flicker light, $\%$ x second & 23.8 & 105.9 & $345 \%$ & $* 0.06$ \\
\hline
\end{tabular}

$\%$ change $=($ post-pre $) /$ pre $\times 100 \% ;$ All veins $=$ all vein branches in retinal image $;$ central veins $=$ first and second branches; peripheral veins $=$ third and fourth branches; two-sided paired $t$-test $p$-values; ${ }^{*}=$ trend of significance. The sample size $n=3$ is small because (i) very small venules are only located at the outer edge of the retina, and their often faint contrast (due to lack of blood supply?) limits DVA-signal detection. 
NIBS improved vision in both patients, and patient K.H. improved in almost all subtests of a cognitive testing battery, some of which recovered up to $40-60 \%$. In this patients vascular dysregulation was found to markedly improved, with increased dilation dynamics in both the artery and vein which was $>300 \%$ above baseline in the smallest microvessels. Both of our patients went back to work after therapy. Thus, the ability of NIBS to trigger recovery of blood flow and improve neurological function is an exciting new option to help long-COVID patients regain their mental functions, an issue which is of growing concern.

\subsection{COVID-19 and vision impairment}

Regarding visual dysfunctions, there are several reports of ocular and vision symptoms in COVID-19. A systematic review and meta-analysis of 16 studies by Aggarwal et al. (2020) with a total of 2,347 cases revealed a prevalence of ocular surface impairments in the order of $11.6 \%$. Here, the most frequently reported ocular manifestations were ocular pain, discharge, redness and follicular conjunctivitis. Indeed, viral RNA was detected in ocular specimens, and mild conjunctivitis can be the first and often only symptom of COVID-19 (Bertoli et al., 2020). LaniLouzada et al. (2020) reported three patients with vascular retinal changes, and among 14 deceased patients three had viral RNA of SARS-CoV-2 in their retina (Casagrande et al., 2020); in fact, retinal ACE2 receptors are used by the coronavirus to enter the cells (Landecho et al., 2021). Furthermore, $22 \%$ of asymptomatic COVID-19 patients had retinal microangiopathy, manifested by cotton wool spots, (Landecho et al., 2021), and they had lower macular vessel density and perfusion density as imaged by optical coherence tomography angiography (OCTa) (Guemes-Villahoz et al., 2021). A few cases of COVID-19 also showed acute macular neuroretinopathy (AMN), a rare condition with intraretinal, reddish-brown macular regions, which are typically wedge-shaped, paracentral scotomas pointing toward the fovea and which were also reported in many young woman that had histories of an influenza-like illness or symptoms (Bhavsar et al., 2016, Ashfaq et al., 2021). Several single cases were reported with paracentral scotoma after infection with SARS-CoV-2 (Virgo et al., 2020) or the English variant of COVID-19 (Macé et al., 2021; see also Masjedi et al., 2019).
Even vaccination can trigger symptoms, and this is not all that surprising given that immune-mediated reactions to vaccines are rather common (Bøhler et al., 2021). Likewise, there are reports of some cases with visual impairments shortly after vaccination: a patient with no history of ocular pathology or migraine reported sudden darkening of his visual field (subjective reduction of visual acuity with visual distortion) three days after his second Pfizer-BioNTech COVID-19 vaccination (Santovito et al., 2021). Similarly, two women developed AMN paracentral scotomas shortly after AstraZeneca vaccination (Mambretti et al., 2021); a 27-year-old woman with unremarkable medical history developed AMNlike paracentral scotomas in one eye after her first AstraZeneca vaccination (Bøhler et al., 2021); a 21-year-old woman with bilateral binocular AMN paracentral scotomas appearing 3 days after receiving her first AstraZeneca vaccination (Book et al., 2021), and AMN symptoms in a 20-year-old-female developing bilateral paracentral scotomata two days after a Pfizer-BioNTech COVID-19 vaccine (Valenzuela et al., 2021).

\subsection{COVID-19 and cognition}

Besides the more subtle vision symptoms, long-COVID patients can develop much more serious neurological symptoms such as cognitive impairments of attention and executive functions. Hampshire et al. (2021) analyzed over 12.000 suspected COVID-19 patients and showed that 386 $(=3,04 \%)$ recovered from early symptoms but later developed significant cognitive deficits as assessed by the web-based Great British Intelligence Test. Compared to less affected patients, the more affected cohort had more cases with cognitive impairments (Liu et al., 2021). In a cohort of hospitalized COVID19 patients cognitive symptoms were reported to be most pronounced, with 59\%-65\% experiencing significant cognitive impairments within four months with marked neurological dysfunctions such as verbal learning skills and executive functions. Long-COVID patients can also suffer from abnormalities in mood functions, including depression, anhedonia and lower stress resilience (Lamontagne et al., 2021). The recognition that severe lung dysfunction can lead to lower brain oxygen levels (Miskowiak et al., 2021) and bilateral hypometabolism in multiple brain regions (Guedj et al., 2021) points our way to possible mechanisms of the neurological symptoms. 


\subsection{COVID-19, vascular regulation and blood flow}

Indeed, a key hallmark of COVID infection and neuronal dysfunction is dysregulated blood flow as recent brain imaging studies attest. Hypometabolism was described by Hosp et al. (2021) in frontoparietal brain regions and by Guedj et al. (2021) in bilateral rectal/orbital gyrus and the right temporal lobe, amygdala and hippocampus with an extension to the right thalamus. These blood flow changes were associated with different symptoms such as hyposmia, anosmia, pain, insomnia, memory and other cognitive impairments. Given these global metabolic changes in the brain it may be that the underlying mechanism of visual and cognitive impairment is not only of neuronal but possibly also of vascular origin. Before discussing the neurovascular mechanism of recovery, we shall summarize what can be learned from our observations and discuss the findings in the context of the existing literature.

\subsection{NIBS and recovery of vision}

The medical benefits of NIBS are not new. Noninvasive electric current stimulation was used long ago for the treatment of different brain diseases. While method of "electrotherapy" was introduced in the 1830s and popular throughout the 19th century (Erb, 1882), its practice was later discontinued. In the last two decades, however, we witness a revival of electrical stimulation as a "novel" means to modulate mental functions following the discovery that brain stimulation alters (short-lasting) brain excitability even after stimulation has ended (Nitzsche \& Paulus 2000). Meantime, numerous studies show the benefits of NIBS in different functional domains, including studies of brain plasticity after visual system damage (for a review see Sabel et al., 2020b) and cognitive decline (Teselink et al., 2021). Regarding vision recovery, several studies show that a 10-day tACS stimulation induces vision restoration (visual field improvements): an open-label observational study of 446 patients with optic nerve damage, traumatic brain injury, inflammation or brain tumor (Fedorov et al., 2011), a double-blind, single-center placebocontrolled study (Sabel et al., 2011, Gall et al., 2011), and a multicenter, prospective, double-blind randomized, controlled trial (Gall et al., 2016) showing improved visual fields by $24 \%$ which lasted at least two months. Of note, the extent of improvement was rather variable, and this variability can now be explained in part by personality traits, mental stress and vascular dysregulation (Sabel et al., 2020c). Specifically, patients who suffered vascular dysregulation - as evident by signs and symptoms of the Flammer Syndrome (Konieczka \& Flammer, 2016, Konieczka et al., 2014) - profited most from NIBS. This is of special interest, considering the strong bi-directional interactions between eye, brain and vascular system (for more details see the "braineye-vascular triad", Sabel et al., 2018).

In both of our patients 10-day treatment of NIBS led to measurable improvements. The visual field expanded after treatment - with additional improvement in patient K.H. at follow-up - and patients noticed vision recovery subjectively. Interestingly, in both cases the scotoma was located in the peripheral visual field sector (outer retina) and it is here where the recovery was most pronounced. Because this is also the area where the smallest vessels are located and because our DVA shows small vessels to have the best recovery rate (see below), we conclude that smaller vessels (who have a greater relative surface) might have a greater risk. But they also have the greatest recovery potential. While, we cannot be certain if the vessel dilation deficit and its recovery in patient K.H. is located in the retina and/or brain, we favor the brain for the following reasons: (i) the symmetric, heterotypic topography of the upper nasal visual field location ( = lower temporal retina) suggests that tissue at or near the chiasma is dysfunctional, and (ii) electric current injecting to the brain via the forehead travels not only to the eyes but also along the optic nerve and the chiasma, passing along the base of the brain (Sabel et al., 2021).

\subsection{NIBS and recovery of cognition}

Besides suffering low vision, both or our patients suffered serious cognitive deficits from which they recovered significantly, though not completely, at the end of the therapy. The efficacy of the treatment on the subjective and objective level was surprisingly fast and relevant in everyday life. Both patients reported to function better in daily life, being able to converse and manage multitasks again, and both were able to go back to work shortly after completing therapy.

Unlike in the visual system, we cannot directly relate retinal vessel responses to functional improvements. Yet, regarding brain cognition, it is reasonable to argue that, like in the retina, the treatment has the most pronounced effect on smaller brain vessels. After all, retinal tissue is ontogenetically very similar 
to, or identical with, brain tissue. While we need to await larger sample studies, we predict that vasodilation recovery in the retina should also correlate with cognitive recovery.

That NIBS can be used to treat cognitive impairments is also not new. For example, anodal tDCS treatment of Alzheimer patients or persons affected by mild cognitive impairment (MCI) leads to shortterm improvements in verbal fluency, especially if combined with cognitive training (Chu et al., 2021). For review, see the meta-analysis by Begemann et al. (2020) of 82 studies ( $n=2784$ patients) showing small but significant improvements of working memory and attention following transcranial magnetic stimulation (TMS) or transcranial Direct Current Stimulation (tDCS) (Begemann et al., 2020, Chu et al., 2021).

\subsection{Vascular recovery: A mechanisms of improvements in vision and cognition?}

Our findings inspired us to speculate about the fundamental recovery mechanisms and the physiological / biological underpinnings of blood flow regulation in long-COVID. Consideration of viral infection and the role of blood flow is therefore needed.

Let us first consider the neurovascular consequences of the virus infection: the SARS-COV 2 viruses can trigger thrombotic events by impacting any or all of the three factors of the "Virchowtriade" (Rudolph Virchow, 1846): (i) changes in the vessel wall or damage to the endothelium, (ii) hypocirculation ("stasis") of the blood, and (iii) hyper-coagulability, i.e. a change in blood composition. Specifically, SARS-COV 2 leads to vascular dysfunction hypoxia and altered capillary transit time (Østergaard et al., 2021). Others found increased levels of acute phase protein with reactive hypergammaglobinaemia which, in turn, increases blood viscosity which negatively impacts hemodynamic properties of the blood (Joob et al., 2021).

In addition, the SARS-COV 2 virus damages the vascular endothelium causing an inflammatory response (endothelitis) with swelling of the endothelial vessel wall and damage to the adjacent pericytes (Libby et al., 2020). This, in turn, causes reduction of the inner diameter of the blood vessels which reduces oxygen and glucose/nutrient delivery to the cells (here: neurons). At a molecular level it is conceivable that the SARS-COV 2 virus activates aryl hydrocarbon receptors (AhRs) and up-regulates diverse AhR-dependent effectors down-stream, resulting in a "Systemic AhR Activation Syndrome" (SAAS) (Turski et al., 2020).

Of note, even small diameter changes can massively influence blood flow: according to a simplified model of the Hagen-Poiseuille equation, the volume flow (blood volume as a function of time) critically depends on the inner radius of the vessel. Therefore, any diameter change alters volume flow by the fourth power (assuming blood pressure is stable). For example, if endothelial cell swelling reduces the radius of a very small vessel to half (all other factors being constant), blood flow is reduced down to $6.25 \%(1 / 16$ !) of its normal value.

If both, blood stasis and diameter reduction are present simultaneously, blood clots in the vascular system are likely. It is evident that such thrombotic events in large vessels can cause immediate clinical symptoms requiring emergency care. However, it is easily conceivable that functional impairments can also be caused by smaller micro-vessel diameter at or near the capillary (unless the number of affected micro-vessels is small). The effect can be capillary congestion, reducing or arresting oxygen supply in the local tissue. Because neural function is particularly sensitive to oxygen deprivation - neuronal processing of action potentials is very fast with local energy peaks -, vascular dysregulation can trigger neurological deficits in different domains (vision, hearing, cognition, etc.). In fact, vascular dysregulation is not limited to neural tissue but it can also affect many other organs (Golubnitschaja, 2019). In sum, it is evident that vascular dysfunction impacts neuronal functioning, but what is the role of blood vessel health and auto-regulation in recovery?

\subsection{Neurovascular mechanisms of recovery of function}

To get answers to this question, we need to understand why our two COVID-19 cases (and possibly patients with other visual or neuropsychiatric disorders) recovered so dramatically and in such a short time period. We propose the following neurovascular mechanisms: in response to neuronal activation (here: induced by visual flicker stimulation in the DVA), COVID veins in the retina and brain fail to dilate due to swelling of the vessel walls, possibly in conjunction with muscle contraction (due to mental stress?). This creates an upstream obstruction of the capillaries, reducing blood flow, depriving neurons of oxygen, reducing their metabolism and functionally inactivating them. Such "silenced" neu- 
rons are too healthy to die, but they can no longer fire action potentials at the required frequency or synchronization, or they are unable to fire neural signals at all, entering into a "hibernation-like" mode. Of course, complete spasm of the arteries or vein stops blood flow altogether, and neurons will inevitably die. Inspired by a syndrome first described by Joseph Flammer (Konieczka \& Flammer 2016), we propose that neuronal inactivation ("silenced" neurons) is caused by venous and arterial vascular dysregulation, where the loss of venous capacity is probably the more relevant one. This metabolic silencing of neurons is perhaps the most fundamental mechanism of neural dysfunction (other than degeneration), but unlike degeneration - reactivation is a possible explanation of both, spontaneous and treatment-induced recovery of function.

One approach to reverse neuronal silencing is noninvasive electric stimulation. Currents delivered to the skull pass to the eyes and cross the skull bone to the frontal cortex. In addition, current passes through the optic nerve foramen to the brain (Sabel et al. 2021). As we showed with a fast visual reaction time paradigm, NIBS can modulate brain functional connectivity networks on a global brain level by way of stimulating different regions including frontal and temporal regions, midbrain, brain-stem and cerebellum (Wu \& Sabel 2021). We propose the following causal chain of events: repeated current injections help both vein (and artery) muscles to relax, leading to artery and vein dilation, re-establishment of blood flow, normalization of oxygen/nutrient delivery, and reactivation of "silent neurons", thus enabling recovery of vision and cognition.

According to Flammer (for review, see Golubnitschaja, 2019), especially the venous system changes are most relevant in vascular dysregulation. When the internal pressure in a vein vessel is reduced following dilation (in our COVID-case even above-normal levels), the vein - due to its greater compliance when pressure is lifted from the venous muscles - now has a greater ability to absorb the rising volume when an increased neuronal activity demands more oxygen from the upstream artery though potassium sensitive sensors in the vessel wall, upon which the artery dilates to increase blood flow (Longden et al., 2017). Venous vessels are able to change the tone of their vascular muscles and thus adjust compliance. If the intravascular pressure on the arterial side of the capillary bed rises more sharply and falls more sharply on the postcapillary venous side than before, under otherwise constant conditions a greater pressure gradient builds up between the precapillary and post-capillary vessels. This increased pressure difference is possibly responsible for the fact that capillary areas that were previously not (or insufficiently) perfused now provide adequate perfusion, increasing oxygen and nutrient supply. This would allow the "silenced" neurons to become reactivated again. We wish to call this the "neurovascular coupling theory of recovery of function".

Our single-case observation is compatible with this hypothesis. We propose that NIBS therapy normalized COVID-induced vascular dysregulation by way of a double-punch approach of forcing neurons to fire action potentials while - at the same time - dilating the respective vessel walls.

While this proposal requires further research and systematic study, this mechanism of action could explain re-oxygenation and reactivation (awakening) of previously "silenced" neurons and thus sustain recovery of function of vision and cognition in COVID-19 patients.

\section{Conclusion}

As we showed, NIBS can improve sensory and cognitive deficits in individuals suffering from long-COVID. Because NIBS improves vascular homeostasis by normalizing arterial and vein perfusion, we propose that NIBS improves metabolic supply of neurons which were disturbed by vasoconstriction. Because recovery of visual and cognitive functions was associated with restoration of vascular health, we further propose that (i) hypometabolic neurons are the probable biological cause of the neurological deficits manifested as long-COVID symptoms, and (ii) the reactivation of "silent" neurons by reoxygenation is the basis of recovery. This conclusion is compatible with our earlier proposal that residual neuronal reserves can be reactivated by behavioral training or NIBS, providing a biological substrate for restoration of central nervous system function in the eye and brain (Sabel et al., 2011). Controlled clinical trials are now needed to confirm our conclusion.

\section{References}

Aggarwal, K., Agarwal, A., Jaiswal, N., Dahiya, N., Ahuja, A., Mahajan, S., ...\& Gupta, V. (2020). Ocular surface manifestations of coronavirus disease 2019 (COVID-19): A systematic review and meta-analysis. PLoS One, 15(11), e0241661. 
Ashfaq, I., Vrahimi, M., Waugh, S., Soomro, T., Grinton, M.E., Browning, A.C. (2021). Acute Macular Neuroretinopathy Associated with Acute Influenza Virus Infection. International Ocular Inflammation Society, 29(2), 333-339.

Bertoli, F., Veritti, D., Danese, C., Samassa, F., Sarao, V., Rassu, N., ...\& Lanzetta P. (2020). Ocular Findings in COVID19 Patients: A Review of Direct Manifestations and Indirect Effects on the Eye. Journal of Ophthalmology, ecollection 4827304.

Begemann, M.J., Brand, B.A., Curčić-Blake, B., Aleman, A. \& Sommer, I.E. (2020). Efficacy of non-invasive brain stimulation on cognitive functioning in brain disorders: a meta-analysis. Psychological Medicine, 50(15), 2465-2486.

Bhavsar, K.V., Lin, S., Rahimy, E., Joseph, A., Freund, K.B., Sarraf, D. \& Cunningham, E.T. (2016). Jr. Acute macular neuroretinopathy: A comprehensive review of the literature. Survey Ophthalmology, 61(5), 538-65.

Bøhler, A.D., Strøm, M.E., Sandvig, K.U., Moe, M.C. \& Jørstad, Ø.K. (2021). Acute macular neuroretinopathy following COVID-19 vaccination. Eye (Lond), 1-2. doi: 10.1038/s41433-021-01610-1.

Bola, M., Gall, C., Moewes, C., Fedorov, A., Hinrichs, H. \& Sabel, B.A. (2014). Brain functional connectivity network breakdown and restoration in blindness. Neurology, 83(6), 542-551.

Bola, M. \& Sabel, B.A. (2015a) Dynamic reorganization of brain functional networks during cognition. Neuroimage, 114, 398-413.

Bola, M., Gall, C. \& Sabel, B.A. (2015b) Disturbed temporal dynamics of brain synchronization in vision loss. Cortex, 67, 134-146.

Book, B.A.J., Schmidt, B. \& Foerster, A.M.H. (2021). Bilateral Acute Macular Neuroretinopathy After Vaccination Against SARS-CoV-2. JAMA Ophthalmolology, 139(7), e212471.

Buso, G., Becchetti, C. \& Berzigotti, A. (2021) Acute splanchnic vein thrombosis in patients with COVID-19: A systematic review. Digestive and Liver Disease, 53(8), 937-949.

Casagrande, M., Fitzek, A., Püschel, K., Aleshcheva, G., Schultheiss, H.P., Berneking, L., Spitzer, M.S. \& Schultheiss, M. (2020). Detection of SARS-CoV-2 in Human Retinal Biopsies of Deceased COVID-19 Patients. Oculular Immunology Inflammation, 28(5), 721-725.

Chu, C.S., Li, C.T., Brunoni, A.R., Yang, F.C., Tseng, P.T., Tu, Y.K., .. \& \& Liang, C.S. (2021). Cognitive effects and acceptability of non-invasive brain stimulation on Alzheimer's disease and mild cognitive impairment: a component network meta-analysis. Journal of Neurology, Neurosurgery and Psychiatry, 92(2), 195-203.

Drüke, D, Pleyer, U., Hoerauf, H., Feltgen, N. \& Bemme, S. (2021). Acute macular neuroretinopathy (AMN) following COVID19 vaccination, American Journal of Ophthalmology Case Report, 24, 101207.

Erb, W. (1882). Handbuch der Elektrotherapie. Verlag V.C.W. Vogel; Leipzig.

Fedorov, A., Jobke, S., Bersnev, V., Chibisova, A., Chibisova, Y., Gall, C., \& Sabel, B. A. (2011). Restoration of vision after optic nerve lesions with noninvasive transorbital alternating current stimulation: a clinical observational study. Brain Stimulation, 4(4), 189-201.

Gall, C., Schmidt, S., Schittkowski, M.P., Antal, A., Ambrus, G.G. Paulus, W., Dannhauer, M., ...\& Sabel, B.A. (2016). Alternating current stimulation for vision restoration after optic nerve damage: a randomized clinical trial. PLoS One, 11, e0156134.

Gall, C., Sgorzaly, S., Schmidt, S., Brandt, S., Fedorov, A., \& Sabel, B. A. (2011). Noninvasive transorbital alternating current stimulation improves subjective visual functioning and vision related quality of life in optic neuropathy. Brain Stimulation, 4(4), 175-188.

Gall, C., Schmidt, S., Schittkowski, M. P., Antal, A., Ambrus, G. G., Paulus, W.,... \& Sabel, B.A. (2016). Alternating Current Stimulation for Vision Restoration after Optic Nerve Damage: A Randomized Clinical Trial. PLoS One, 11(6), e0156134.

Garhofer, G., Bek, T., Boehm, A.G., Gherghel, D., Grunwald, J., Jeppesen, P., .. \& \& Schmetterer, L. (2010). Use of the retinal vessel analyzer in ocular blood flow research. Acta Ophthalmologica, 88(7), 717-722.

Golubnitschaja, O. 2019 (Ed.) "Flammer Syndrome: From Phenotype to Associated Pathologies, Prediction, Prevention and Personalisation", Springer, Berlin, Germany.

Guedj, E., Campion, J.Y., Dudouet, P., Kaphan, E., Bregeon, F., Tissot-Dupont, H., ...\& E Eldin C. (2021). ${ }^{18}$ F-FDG brain PET hypometabolism in patients with long COVID. European Journal of Nuclear Medicine and Molecular Imaging, 48(9), 2823-2833.

Guemes-Villahoz, N., Burgos-Blasco, B., Vidal-Villegas, B., Donate-López, J., de la Muela, M.H., López-Guajardo, L., Martín-Sánchez, F.J., García-Feijoó, J. (2021). Reduced macular vessel density in COVID-19 patients with and without associated thrombotic events using optical coherence tomography angiography. Graefes Archive for Clinical and Experimental Ophthalmology, 259(8), 2243-2249.

Gugleta, K., Kochkorov, A., Waldmann, N., Polunina, A., Katamay,R., Flammer,J. \& Orgual, S. (2012). Dynamics of retinal vessel response to flicker light in glaucoma patients and ocular hypertensives. Graefes Archive for Clinical and Experimental Ophthalmology 250(4), 589-594.

Hampshire, A., Trender, W., Chamberlain, S.R., Jolly, A.E., Grant, J.E., Patrick, F., . . \& Mehta, M.A. (2021). Cognitive deficits in people who have recovered from COVID-19. EClinicalMedicine, 39, 101044.

Hosp, J.A., Dressing, A., Blazhenets, G., Bormann, T., Rau, A., Schwabenland, M., ...\& Meyer, P.T. (2021). Cognitive impairment and altered cerebral glucose metabolism in the subacute stage of COVID-19. Brain, 144(4), 1263-1276.

Joob, B. \& Wiwanitkit, V. (2021). Blood viscosity of COVID19 patient: a preliminary report. American Journal of Blood Research, 11(1), 93-95.

Kang, N., Lee, R.D., Lee J.H. \& Hwang M.H. (2020). Functional balance and postural control improvements in patients with stroke after noninvasive brain stimulation: a meta-analysis. Archives of Physical Medicine and Rehabilitation, 101(1), 141-153.

Kisely, S., Li, A., Warren, N., \& Siskind, D. (2018). A systematic review and meta-analysis of deep brain stimulation for depression. Depression and Anxiety, 35(5), 468-480. 
Konieczka, K. \& Flammer, J. (2016). Phenomenology and Clinical Relevance of the Flammer Syndrome. Klinische Monatsblätter für Augenheilkunde, 233, 1331-1336.

Konieczka, K., Ritch, R., Traverso, C.E., Kim, D.M., Kook, M.S., Gallino, A., Golubnitschaja, O., Erb, C., Reitsamer, H.A., Kida, T., Kurysheva, N. \& Yao, K. (2014). Flammer syndrome. EPMA Journal, 5, eCollection.

Landecho, M.F., Yuste, J.R., Gándara, E., Sunsundegui, P., Quiroga, J., Alcaide, A.B. \& García-Layana, A. (2021). COVID-19 retinal microangiopathy as an in vivo biomarker of systemic vascular disease? Journal of Internal Medicine, 289(1), 116-120.

Lamontagne, S.J., Winters, M.F., Pizzagalli, D.A. \& Olmstead, M.C. (2021). Post-acute sequelae of COVID-19: Evidence of mood \& cognitive impairment. Brain Behavior \& Immunity Health, 17, 100347.

Lani-Louzada, R., Ramos, C.D.V.F., Cordeiro, R.M. \& Sadun, A.A. (2020). Retinal changes in COVID-19 hospitalized cases. PLoS One, 15(12), e0243346.

Libby, P. \& Lüscher, T. (2020). COVID-19 is, in the end, an endothelial disease. European Heart Journal, 41(32), 3038-3044.

Liu, Y.H., Wang, Y.R., Wang, Q.H., Chen, Y., Chen, X., Li, Y., ...\& Wang, Y.J. (2021). Post-infection cognitive impairments in a cohort of elderly patients with COVID-19. Molecular Neurodegeneration, 16(1), 48.

Longden, T.A., Dabertrand, F., Koide, M., Gonzales, A.L., Tykocki, N.R., Brayden, J.E., Hill-Eubanks, D. \& Nelson, M.T. (2017). Capillary $K+$-sensing initiates retrograde hyperpolarization to increase local cerebral blood flow. Nature Neuroscience, 20(5), 717-726.

Macé, T. \& Pipelart, V. (2021). Acute macular neuroretinopathy and SARS-CoV-2 infection: Case report. Journal Français D'Ophtalmologie, 44(9), e519-e521.

Mambretti, M., Huemer, J., Torregrossa, G., Ullrich, M., Findl, O. \& Casalino, G. Acute Macular Neuroretinopathy following Coronavirus Disease 2019 Vaccination. (2021). Oculular Immunology and Inflammation, 29(4), 730-733.

Masjedi, M., Pourazizi, M. \& Hosseini, N.S. (2021). Acute macular neuroretinopathy as a manifestation of coronavirus disease 2019: A case report. Clinical Case Reports, 9, e04976.

Miskowiak, K.W., Johnsen, S., Sattler, S.M., Nielsen, S., Kunalan, K., Rungby, J., Lapperre, T. \& Porsberg, C.M. (2021) Cognitive impairments four months after COVID-19 hospital discharge: Pattern, severity and association with illness variables. European Neuropsychopharmacology, 46, 39-48.

Nitsche, M.A. \& Paulus, W. (2000). Excitability changes induced in the human motor cortex by weak transcranial direct current stimulation. The Journal of Physiology, 527, 633-9.

Nurek, M., Rayner, C., Freyer, A., Taylor, S., Järte, L., MacDermott, N., Delaney B.C., Delphi panellists. (2021). Recommendations for the recognition, diagnosis, and management of long COVID: a Delphi study. British Journal of General Practice, 71(712), e815-e825.

Østergaard, L. (2021). SARS CoV-2 related microvascular damage and symptoms during and after COVID-19: Consequences of capillary transit-time changes, tissue hypoxia and inflammation. Physiological Reports, 9(3), e14726.
Proal, A.D. \& VanElzakker M.D. (2021), Long COVID or Postacute Sequelae of COVID-19 (PASC): An overview of biological factors that may contribute to persistent symptoms. Frontiers in Microbiology 12, 698169.

Querques, G., Borrelli, E., Sacconi, R., De Vitis, L., Leocani, L., Santangelo, R., ...\& Bandello, F. (2019). Functional and morphological changes of the retinal vessels in Alzheimer's disease and mild cognitive impairment. Scientific Reports, 9(1), 63.

Sabel, B.A., Fedorov, A.B., Naue, N., Borrmann, A., Herrmann, C., \& Gall, C. (2011). Non- invasive alternating current stimulation improves vision in optic neuropathy. Restorative Neurology and Neuroscience, 29(6), 493-505.

Sabel, B.A., Flammer, J.\& Merabet, L.B. (2018). Residual vision activation and the brain-eye-vascular triad: Dysregulation, plasticity and restoration in low vision and blindness a review. Restorative Neurology and Neuroscience, 36, 767-791.

Sabel, B.A., Hamid, A.I.A., Borrmann, C., Speck, O. \& Antal, A. (2020a). Transorbital alternating current stimulation modifies BOLD activity in healthy subjects and in a stroke patient with hemianopia: A 7 Tesla fMRI feasibility study. International Journal of Psychophysiology, 154, 80-92.

Sabel, B.A., Kresinsky, A., Cárdenas-Morales, L., Haueisen, J., Hunold, A., Dannhauer, M. \& Antal, A. (2021). Evaluating current density modeling of non-invasive eye and brain electrical stimulation using phosphene thresholds, IEEE TNSRE, 29, 2133-2141.

Sabel, B.A., Thut, G., Haueisen, J., Henrich-Noack, P., Herrmann, C.S., Hunold, A., . . \& \& Antal, A. (2020b). Vision modulation, plasticity and restoration using non-invasive brain stimulation - An IFCN-sponsored review. Clinical Neurophysiology, 131, 887-911.

Sabel, B.A., Wang, J., Cárdenas-Morales, L., Faiq, M., Heim, C. \& Golubnitschaja, O. (2019). Flammer Syndrome: Psychological Causes and Consequences of Visual Impairment. In: O. Golubnitschaja (Ed.) "Flammer Syndrome: From Phenotype to Associated Pathologies, Prediction, Prevention and Personalisation", Springer, Berlin, Germany, pp. 29-77.

Sabel, B.A., Wang, J., Fähse, S., Cardenas-Morales, L. \& Antal, A. (2020c). Personality and stress influence vision restoration and recovery in glaucoma and optic neuropathy following alternating current stimulation: implications for personalized neuromodulation and rehabilitation. EPMA Journal, 11, 177-196.

Santovito, L.S. \& Pinna, G. (2021). Acute reduction of visual acuity and visual field after Pfizer-BioNTech COVID-19 vaccine 2nd dose: a case report. Inflammation Research, 70(9), 931-933.

Seifert, B.U. \& Vilser, W. (2002). Retinal Vessel Analyzer (RVA) - design and function. Biomedizinische Technik (Berl), 47(Suppl 1) Pt 2, 678-681.

Siegert, A., Diedrich, L. \& Antal, A. (2021) New Methods, old Brains-a systematic review on the effects of tDCS on the cognition of elderly people. Frontiers in Human Neuroscience, 27(15), 730134.

Teselink, J., Bawa, K.K., Koo, G.K., Sankhe, K., Liu, C.S., Rapoport, M., ...\& Lanctôt, K.L. (2021). Efficacy of non-invasive brain stimulation on global cognition and neuropsychiatric symptoms in Alzheimer's disease and mild 
cognitive impairment: A meta-analysis and systematic review. Ageing Research Reviews, 72, 101499.

Westwood, S.J., Radua, J., \& Rubia, K. (2021). Noninvasive brain stimulation in children and adults with attentiondeficit/hyperactivity disorder: A systematic review and meta-analysis. Journal of Psychiatry \& Neuroscience, 46(1), E14-E33.

Wu, Z. \& Sabel, B.A. (2021) Spacetime in the brain: rapid brain network reorganization in visual processing and recovery. Scientific Reports, 11, 17940.

Valenzuela, D.A., Groth, S., Taubenslag, K.J., Gangaputra, S. (2021). Acute macular neuroretinopathy following PfizerBioNTech COVID-19 vaccination. American Journal of Ophthalmology Case Reports, 24, 101200.
Virchow, R. Weitere Untersuchungen über die Verstopfung der Lungenarterien und ihre Folge. Traube's Beiträge experimentelle Patholologie und Physiologie, 1846, 2. Jg., S. 21-31.

Virgo, J. \& Mohamed, M. (2020). Paracentral acute middle maculopathy and acute macular neuroretinopathy following SARS-CoV-2 infection. Eye (Lond), 34(12), 2352-2353.

WHO (download 21 Nov 2021): https://www.who.int/publica tions/i/item/WHO-2019-nCoV-Post_COVID-19_conditionClinical_case_definition-2021.1 